\title{
The role of radiology in the evaluation of the immunotherapy efficacy
}

\author{
Marco Calandri ${ }^{1,2}$, Federica Solitro ${ }^{1,2}$, Valeria Angelino ${ }^{1,2}$, Federica Moretti ${ }^{1,2}$, Andrea Veltri ${ }^{1,2}$ \\ ${ }^{1}$ Radiology Unit, Department of Oncology, University of Torino, Torino, Italy; ${ }^{2}$ A.O.U. San Luigi Gonzaga Hospital, Regione Gonzole, Orbassano \\ (TO), Italy \\ Contributions: (I) Conception and design: M Calandri, F Solitro, A Veltri; (II) Administrative support: None; (III) Provision of study materials \\ or patients: None; (IV) Collection and assembly of data: M Calandri, F Solitro, V Angelino, F Moretti; (V) Data analysis and interpretation: M \\ Calandri, F Solitro, V Angelino, A Veltri; (VI) Manuscript writing: All authors; (VII) Final approval of manuscript: All authors. \\ Correspondence to: Marco Calandri, MD. Department of Oncology, University of Torino, Torino, Italy. Email: marco.calandri@unito.it.
}

\begin{abstract}
In the last years, a great interest has arisen on immunotherapy for the treatment of advanced non-small cell lung cancer (NSCLC). Check-point inhibitor drugs are now considered clinical practice standard in different settings and their use is expected to increase significantly in the near future. As treatment options for lung cancer advance and vary, the different patterns of radiological response increase in number and heterogeneity. To correctly evaluate the radiological findings after and during these treatments is of paramount importance, both in the clinical and sperimental setting. In consideration of their peculiar mechanism, immunotherapies can determine unusual response patterns on imaging, that cannot be correctly evaluated with the traditional response criteria such as World Health Organization (WHO) and Response Evaluation Criteria in Solid Tumours (RECIST). Therefore, during these years, several response criteria [immune-related response criteria (irRC), irRECIST and iRECIST] were proposed and applied in clinical trials on immunotherapies. The aim of this review is to describe the radiological findings after immunotherapy, to critically discuss the different response criteria and the imaging of immune-related adverse events.
\end{abstract}

Keywords: Lung cancer; immunotherapy; radiology; response criteria; adverse events

Submitted May 15, 2018. Accepted for publication May 16, 2018.

doi: $10.21037 /$ jtd.2018.05.130

View this article at: http://dx.doi.org/10.21037/jtd.2018.05.130

\section{Introduction}

In recent years, a better molecular characterization of lung cancer resulted in relevant improvements in treatment planning and led to the creation of effective targeted therapies (1). In general, the term targeted therapy includes a wide range of different therapies, such as inhibitors of signal transduction, inhibitors of angiogenesis or modulators of the immune system (2). Despite non-small cell lung cancer (NSCLC) was initially considered not responsive to immune-mediated drugs because of failed attempts with interleukin-2, interferon or Bacillus of Calmette-Guerin (3), in the last years a great interest has arisen on new and promising immunomodulatory monoclonal antibodies. In fact, since 2015, three immune check-point inhibitors (ICIs) were approved by the US Food and Drug Administration (FDA) for the treatment of advanced NSCLC (4-6) and combinations of different immunomodulatory monoclonal antibodies are under investigation (7). The use of these drugs is spreading worldwide, they are now considered clinical practice standard in different settings and their use is expected to increase significantly in the near future (2).

As treatment options for lung cancer advance and vary, the different patterns of radiological response increase in number and heterogeneity. Specifically, in consideration of their peculiar mechanism, ICIs can determine unusual response patterns (such as the pseudo-progression pattern) and adverse event profiles on imaging. 
The aim of this review is to describe the radiological findings after ICIs therapy, to discuss the specific response criteria [including immune-related response criteria (irRC), irRECIST and iRECIST] and the imaging of immune related adverse events.

\section{Radiological response evaluation}

\section{WHO and RECIST 1.1}

To evaluate the effectiveness of chemotherapy agents, radiologists have traditionally relied on response criteria, such as WHO and RECIST criteria.

WHO criteria were first proposed in 1981; the concept behind them was to evaluate the tumor burden as the sum of the products of the diameters. These criteria were often considered overly sensitive and one of the criticisms usually addressed to them is the fact that progressive disease (PD) could be easily diagnosed on the basis of minor changes in tumor size or even measurement errors (8). RECIST criteria were proposed in 2000 (and revised in 2009, RECIST 1.1) by the WHO, the National Cancer Institute, and the European Organization for Research and Treatment of Cancer (EORTC). The RECIST criteria include definitions of minimum size of lesions and use of a measurement in only one dimension. According to these criteria, PD is defined as the appearance of new lesions or, at least, a $20 \%$ increase in the sum of diameters of target lesions, taking as reference the smallest sum on study (versus an increase of $25 \%$ or more according to the WHO criteria). These criteria have been widely used for the outcome evaluation in prospective studies and trials (9-11).

\section{Morphological changes after immunotherapy}

Relevantly, both these criteria (WHO and RECIST 1.1) assumed that an increase in size of the target lesion and/ or the appearance of new lesions denote a PD in patients treated with cytotoxic agents, which are subsequently discontinued once PD has been demonstrated. Indeed, these response criteria are optimized to evaluate the cytotoxic effects of conventional chemotherapeutic agents on tumors, where shrinkage of the lesion at the cross-sectional imaging is strictly related to a response to cytotoxic drugs $(12,13)$.

Targeted therapies, especially immune-mediated drugs, may determine different patterns of radiological response, not correctly estimated with WHO and RECIST criteria. One of the most common and challenging condition for the morphological evaluation of the response is the so called "pseudo-progression", a condition where the target lesion apparently continues to grow at the first cross sectional imaging (CSI) check (even with concomitant appearance of new lesions) and then remains stable, shrinks in size or disappears during the subsequent CSI controls. Proposed etiologies for these morphologic changes are two: (I) continuous tumor growth during the immune response mounting and/or (II) inflammation of the existing lesions and of other lesions initially not visible at CSI, due to hyper-activated T cells (13). Pseudo-progression has been reported for anti-PD-1, anti-PD-L1, and anti-CTLA-4 agents not only in lung cancer but also in other cancers, such as melanoma, renal cell carcinoma, and bladder cancer (13-15). This response pattern can occur in the lymph nodes but is more commonly described in non-nodal sites $(13,16)$.

Another interesting and atypical pattern of response after immunotherapy is the so-called dissociated response, defined as the concomitant decrease in certain tumoral elements and increase in other sites (Figure 1). According to literature series (17), the incidence of this response pattern is not negligible, and some organs may be more often involved [such as adrenal lesions (18)].

To correctly interpret the changes in morphology of the lesion under ICIs treatment, in 2004 and 2005 a panel of experts summed up in five statements the criticalities of these morphological changes of the tumor $(1,3)$ : (I) time needed to see anti-tumoral effect may be longer for immune therapies than for cytotoxic therapies; (II) responses to immune therapies can be demonstrated even after conventional PD; (III) to discontinue immune therapy cannot be appropriate in some cases, unless PD is confirmed (as is usually done for response); (IV) allowance for "clinically insignificant" PD (e.g., small new lesions in the presence of other responsive lesions) is recommended; and $(\mathrm{V})$ maintained stable disease (SD) may represent presence of antitumor activity.

\section{irRC}

The diffuse awareness of these different radiological patterns, gained from the experience on the first melanoma trials, led to the proposal of the irRC (3). Based from an adaptation of WHO criteria, irRC differs significantly from RECIST 1.1. In fact, the number of lesion to evaluate is higher if compared to RECIST 1.1 (up to 5 per organ, up to 10 visceral $v$ s. 2 per organ, 5 in total) and the 

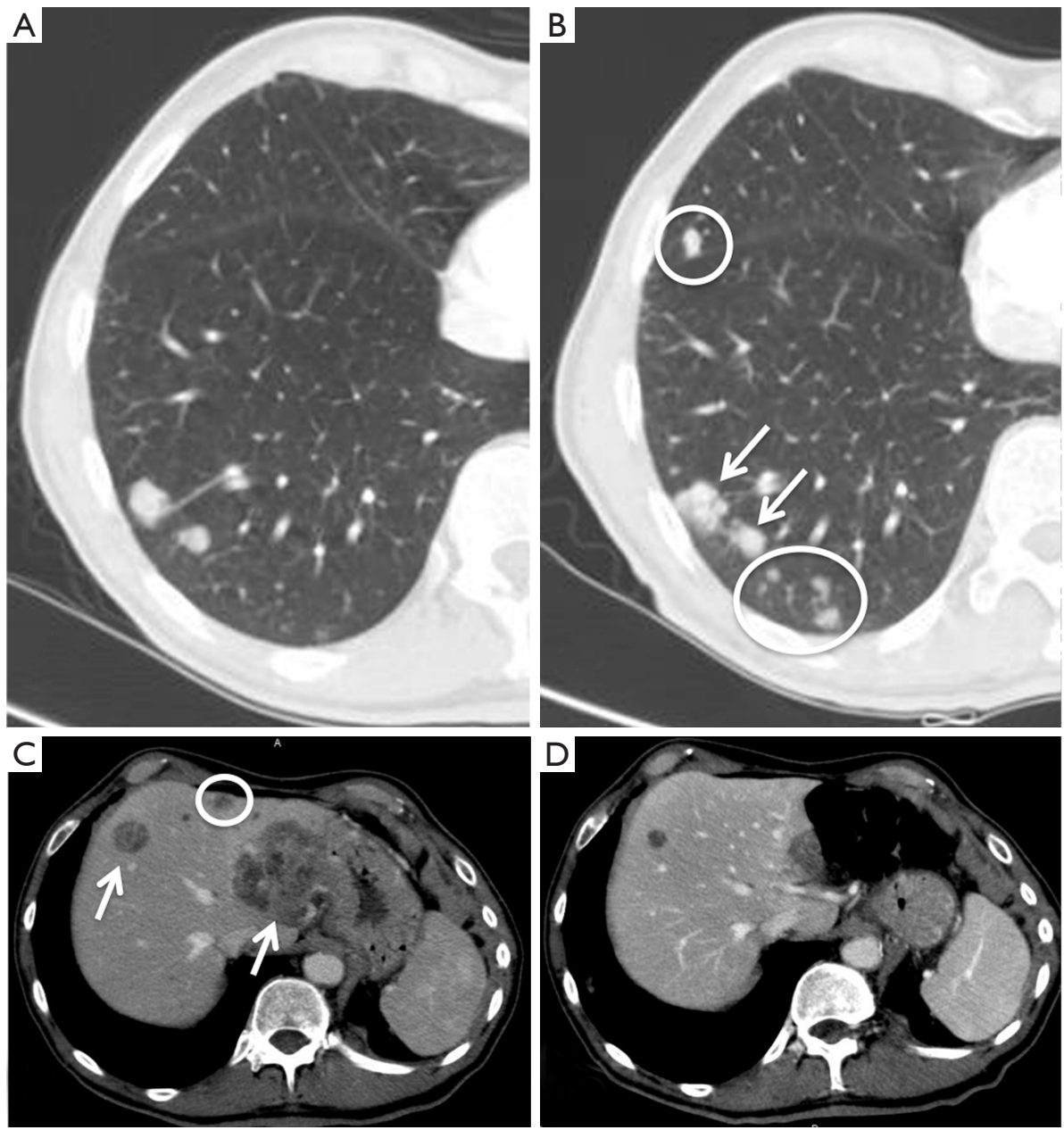

Figure 1 Pseudoprogression and dissociated response. A 68-year-old male patient with history of NSCLC metastatic to lung and liver. (A,B) Axial chest CT images before (A) and after 12 weeks (B) of treatment with nivolumab (an anti-PD-1 monoclonal antibody) showing increase in size of the lesions (arrows) and appearance of new lung nodules (circles: these findings were stable at the subsequent CSI); (C,D) axial CT images of the same patients before (C) and after 12 weeks (D) of treatment showing decrease in size of some liver lesions (arrows) and disappearance of a metastatic lesion (circle). NSCLC, non-small cell lung cancer; CSI, cross sectional imaging.

measurement is bidimensional instead of unidimensional. Relevantly, in irRC, new lesions are not considered PD but are incorporated in total tumor burden (Table 1).

Despite these described advancements, several critiques were addressed to irRC criteria. First, the reproducibility of bidimensional assessment is lower if compared with unidimensional assessment; second, the large number of target lesions to be measured can be time consuming; third, lymph nodes assessment is not clearly evaluated $(1,19)$. In addition, despite some literature data showing underestimation of the benefit of pembrolizumab in $15 \%$ of melanoma patients with RECIST 1.1 criteria if compared with irRC (16), data concerning ICIs and lung cancer patients are less clear: for instance, recent reports evaluating ICIs response in lung cancer patients suggested similar prognostic significance between irRC and RECIST 1.1 criteria (20).

\section{irRECIST}

In order to obtain a more reproducible and faster reporting system, Nishino and Coll. proposed the irRECIST criteria (12), a system based on unidimensional evaluation and lower number of target lesions. irRECIST are very 
Table 1 Comparison of irRC, irRECIST and iRECIST criteria

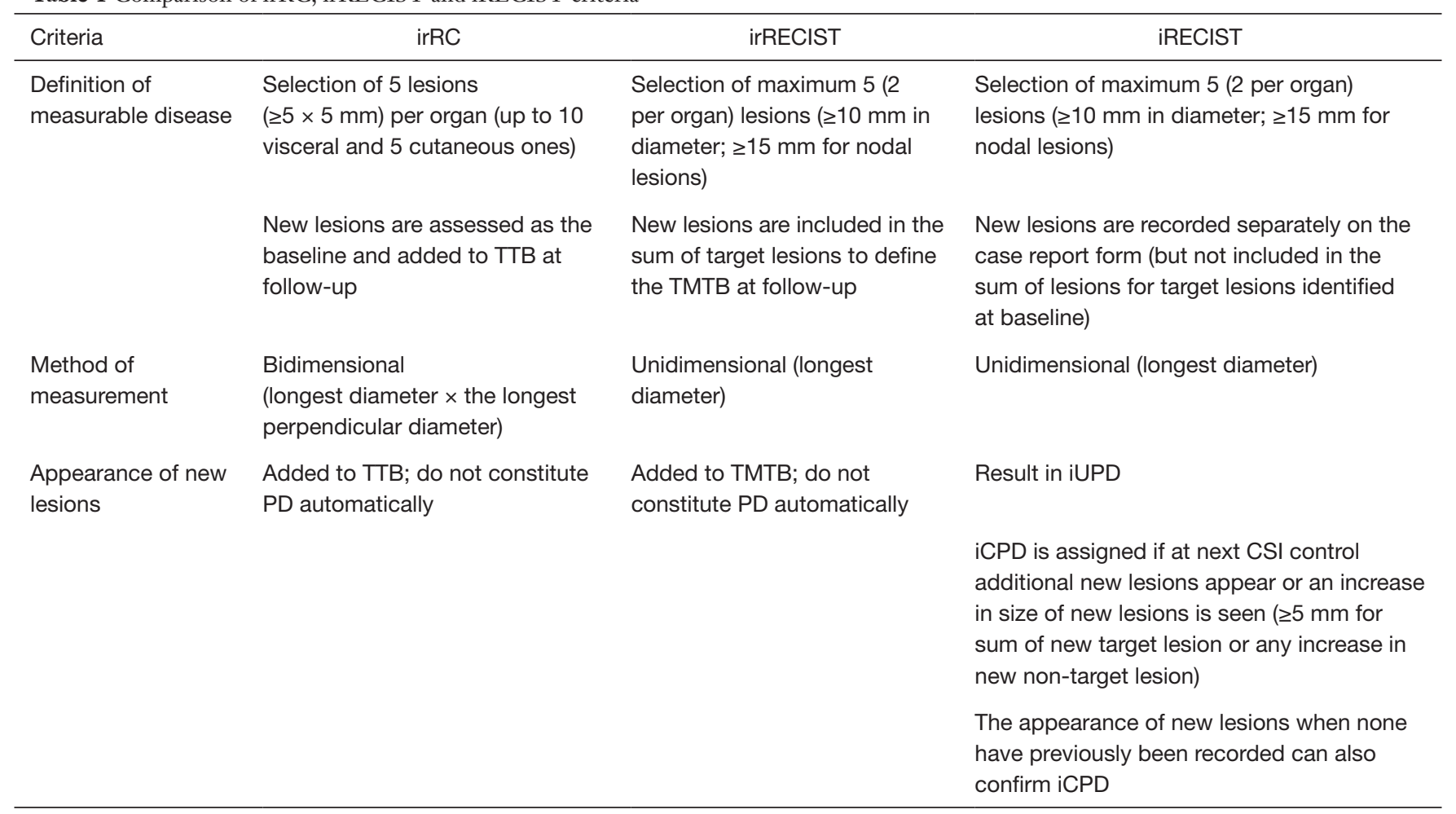

irRC, immune-related response criteria; irRECIST, immune-related Response Evaluation Criteria in Solid Tumors; iRECIST, immune RECIST; TTB, total tumor burden; TMTB, total measured tumor burden; PD, progressive disease; iUPD, unconfirmed progressive disease; iCPD, confirmed progressive disease; CSI, cross-sectional imaging.

similar to RECIST 1.1: in fact, they are similar in terms of criteria for selecting target (5 total target lesions with a maximum of 2 per organ) and non-target lesion, and how these lesions should be measured.

The main difference between irRECIST and RECIST 1.1 is how new lesions are incorporated into the response assessment. In irRECIST, new lesions are incorporated in the total measured tumor burden (TMTB); differently from the RECIST 1.1, new lesions do not immediately mean PD. In irRECIST, new lesions should be defined as measurable or non-measurable, and those selected as new targets should meet the same criteria for inclusion as the baseline lesions. Thus, in case of new target lesions appearance, the longest diameters of existing non-nodal target plus new non-lymph node target lesions, and short-axis diameters of existing lymph node targets plus new lymph node target lesions constitute the TMTB. This method allows to do not discontinue a potentially effective therapy in case of appearance of new lesions.

The specific thresholds for irPR (partial response) and irPD are aligned with RECIST 1.1 (see Table 2). Confirmatory evaluation of PD is not mandatory; however, confirmation of progression should be recommended for patients with a minimal TMTB increase over $20 \%$, particularly during the first 12 weeks of treatment $(12,19)$.

\section{iRECIST}

Recently, the RECIST working group prospectively analyzed data from several clinical trials of immunotherapeutic agents (18), finding out a diffused use of RECIST 1.1 to define primary and secondary efficacy endpoints whereas irRC and irRECIST were used for exploratory/minor endpoints only $(16,21)$. Critiques were also addressed to the substantial heterogeneity of the response criteria used in different clinical trials and subsequent potentially misleading interpretation of pooled datasets. The RECIST working group therefore came to propose the iRECIST criteria with the goal to provide a consistent and common language for the management of data deriving from 
Table 2 Comparison of irRC, irRECIST and iRECIST response assessment

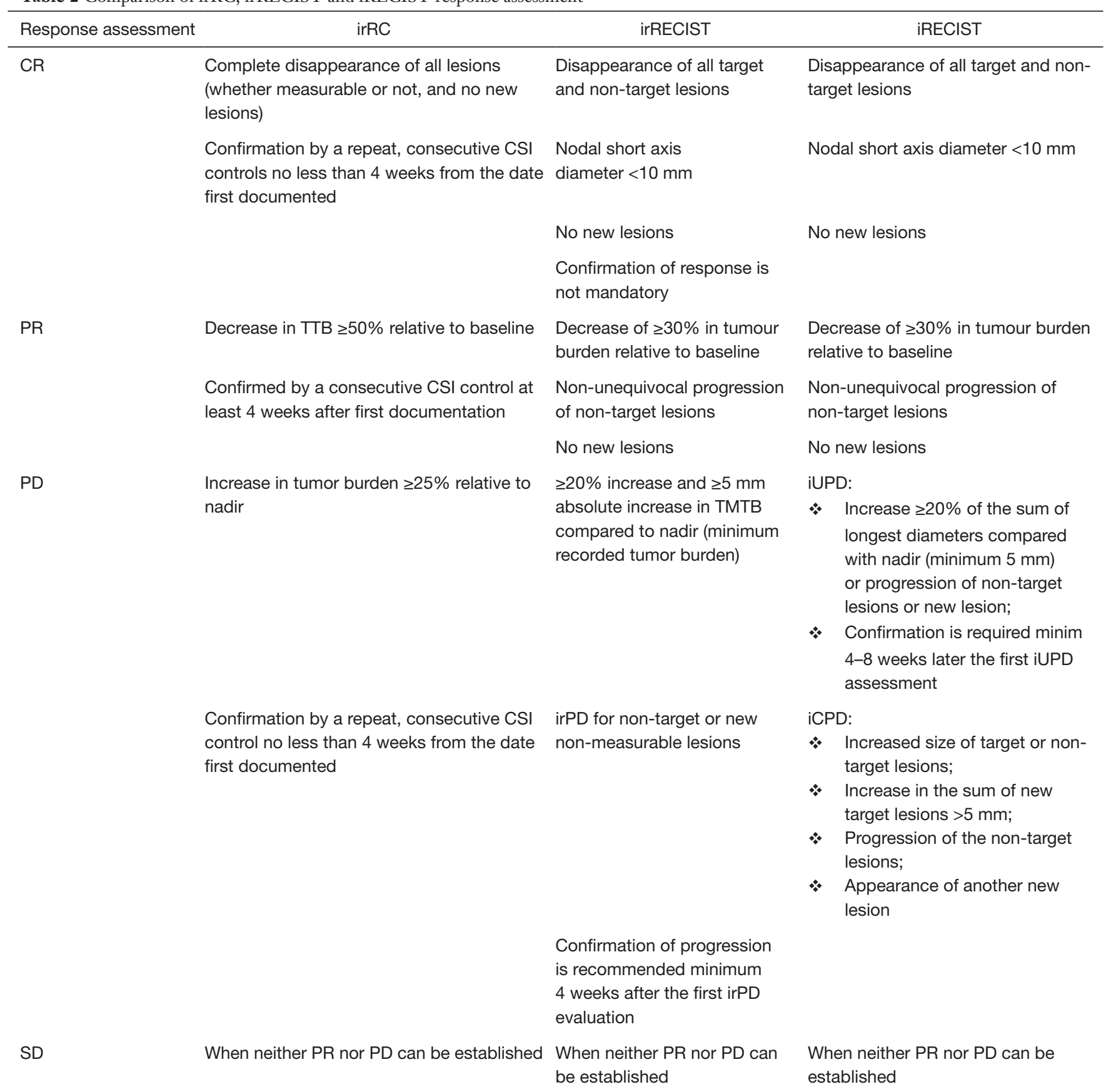

irRC, immune-related response criteria; irRECIST, immune-related Response Evaluation Criteria in Solid Tumors; iRECIST, immune RECIST; CR, complete response; PR, partial response; PD, progressive disease; SD, stable disease; CSI, cross-sectional imaging; TTB, total tumor burden; TMTB, total measured tumor burden; iUPD, unconfirmed progressive disease; iCPD, confirmed progressive disease.

different trials of immunotherapeutic agents (21).

iRECIST are comparable with RECIST 1.1 and irRECIST in terms of recommended imaging modalities, definitions of measurable lesions and target lesions $(9,18)$.
Notably, target and non-target lesions are not counted together and therefore not added to the greatest dimensions of all target lesions, in contrast to irRECIST (see Table 1).

The response categories of iRECIST include iCR 
(complete response), iSD (stable disease) and iPR (partial response) but also unconfirmed PD (iUPD) and confirmed PD (iCPD) (see Table 2). These last two categories enable an improved characterization of atypical responses. Indeed, taking into account target lesions, $\mathrm{iCR}$, iPR, and iSD are still possible after iUPD shows up, as long as iCPD is not identified. Furthermore, the demonstration of new lesions is classified as iUPD. To be assessed as iCPD, a further increase in size of previous new lesions $(5 \mathrm{~mm}$ for the sum of target lesions or any increase in non-target lesions) or additional new lesions appearance is required at the subsequent CSI.

Of note, the RECIST work group suggest, once iUPD is identified, to perform the subsequent CSI evaluation between 4 and 8 weeks after, in order to allow the continuity of the treatment but also to enable a salvage therapy, if needed.

In a recent retrospective analysis Tazdait et al. (17) confirmed the usefulness of iRECIST criteria, showing $11 \%$ of atypical responses, otherwise underestimated by the RECIST 1.1.

\section{Radiological evaluation of adverse events}

Immune-related adverse events (irAE) differ relevantly from adverse events of cytotoxic agents or targeted therapies. Radiologists should therefore be aware of the existence of these adverse events and of their radiological features in order to obtain an early diagnosis and a prompt treatment (22).

According to the literature, incidence is not negligible and varies between the $26 \%$ of anti-PD-1 drugs and $13.7 \%$ of anti-PD-L1 drugs (23), while pembrolizumab, a PD-1 inhibitor, shows a lower rate of severe-to-life threatening adverse events in comparison with ipilimumab in advanced melanoma trial (24); ipilimumab (anti-CTLA4 drug) has been recently proposed in association with nivolumab (antiPD1) in NSCLC phase I trial, showing a percentage of adverse events of $17.4 \%$ and a treatment related deaths of $1.2 \%$ (seven patients) (25).

A great number of organs and tissues can be involved in irAEs: skin and mucosae, bowels, lung, liver, pancreas, glands (thyroid, hypophysis, adrenal glands), kidney, eyes, central nervous system, blood, muscles and bones (25).

The most common irAEs for which imaging is mandatory are colitis (7-17\% incidence) and hypophysitis (4-11\%) with the anti-CTLA-4 monoclonal antibody ipilimumab, and pneumonitis (3-6\%) with anti-PD-1 agents $(13,23,26-28)$.
Colitis typically occurs after 6-7 weeks from the beginning of anti-CTLA-4 monoclonal antibody treatment and generally the colorectal involvement is diffuse $(13,25)$. CT shows a fluid-filled colon, with thickened wall and increased mucosal enhancement, and mesenteric hyperemia. Sometimes the wall thickening can be segmental and superimposed to diverticulitis, the so called "segmental colitis associated with diverticulosis" (2).

Rarely, colitis can lead to perforation, easily identified by plain radiography or non-contrast CT (13).

Patients with anti-CTLA-4 monoclonal antibody hypophysitis presented with different non-specific symptoms, in particular headache and fatigue, and develop an anterior hypopituitarism persistent at follow-up. Magnetic resonance (MR) imaging demonstrate diffuse pituitary enlargement and/or heterogeneous pituitary enhancement at post-contrast images. Pituitary enlargement is generally reversible after glucocorticoid treatment.

Pneumonitis occur in 2-6\% of Patients treated with antiPD-1/PDL-1 or CTLA-4 antibodies (25). According to Nishino et al. (29), median time to onset of pneumonitis is 2.8 months (range, 9 days-19.2 months), but in some cases recurrent pneumonitis ("pneumonitis flare") were diagnosed months after a successful steroid treatment of the irAEs, with or without retreatment with the immunotherapy previously discontinued.

CT scans generally demonstrate ground-glass opacities, consolidations, bronchiectasis, interlobular septal thickening and intralobular lines (30). Sometimes CT shows crazy-paving pattern also, with diffuse or localised lung involvement (Figures 2-4).

Four CT patterns have been recently identified in patients with nivolumab-induced pneumonitis (29): cryptogenic organizing pneumonia (COP) pattern in $65 \%$ of patients, nonspecific interstitial pneumonia pattern in $15 \%$, hypersensitivity pneumonitis pattern in $10 \%$, and acute interstitial pneumonia (AIP)/acute respiratory distress syndrome (ARDS) in 10\%.

Therefore, CT examinations show: (I) patchy groundglass opacities and consolidation in a subpleural, peribronchial or band pattern, sometimes accompanied by the reversed halo sign in the COP pattern; (II) bilateral ground-glass opacities with reticular opacities, traction bronchiectasis or bronchiolectasis and minimal or absent honey-combing in a basal distribution with subpleural sparing in the nonspecific interstitial pneumonia pattern; (III) centrilobular nodules and mosaic attenuation due to air trapping with an upper lobe-predominant distribution in 


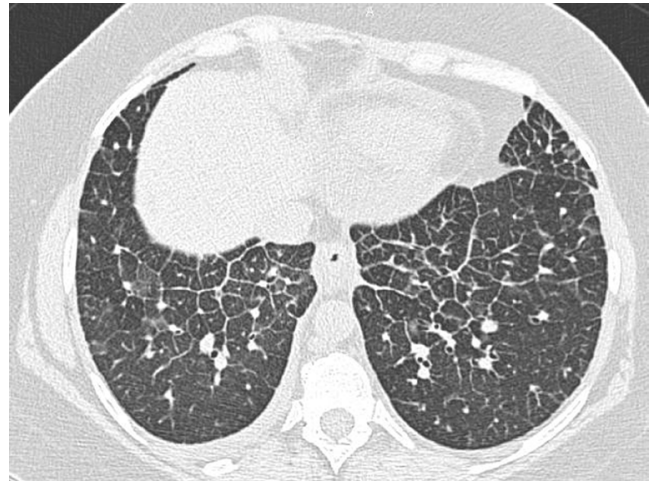

Figure 2 A 42-year-old female patient with NSCLC in treatment with nivolumab (anti-PD-1 monoclonal antibody). Axial chest CT reveals interlobular septal thickening with small patchy groundglass opacities in the lower lobes, in a crazy-paving configuration. NSCLC, non-small cell lung cancer.

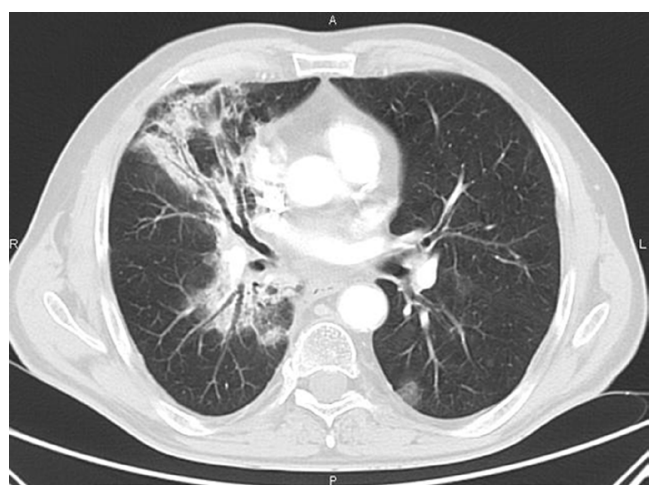

Figure 3 A 64-year-old male patient with NSCLC in treatment with pembrolizumab (anti-PD-L1 monoclonal antibody). Axial chest CT shows pneumonitis characterized by peri-bronchial confluent parenchymal consolidation in the right lung in association with bilateral ground-glass opacities. NSCLC, nonsmall cell lung cancer.

case of hypersensitivity pneumonitis; (IV) patchy bilateral ground-glass opacities with consolidation in the dependent lung in the AIP/ARDS pattern.

Patients on immune checkpoint inhibitors can also develop clinically silent new imaging findings, such as sarcoid-like distributed lymph nodes (bilateral hilar and mediastinal) (2). These nodes can't be distinguished from nodal metastases, but in the setting of response at other sites their distribution resembles that of sarcoidosis. They might regress spontaneously, so treatment should not be

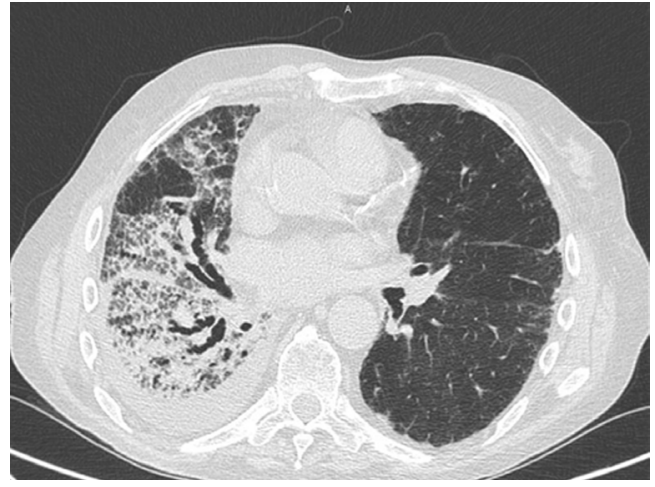

Figure 4 A 78-year-old male patient with NSCLC in treatment with nivolumab (anti-PD-1 monoclonal antibody). Axial chest CT demonstrates pneumonitis characterized by fibrosing reticulation, intralobular thickening and traction bronchiectasis, with large areas of honeycombing in the right lung. Pleural effusion is associated. In the left lung there is minimal subpleural interstitial reticulation. NSCLC, non-small cell lung cancer.

discontinued.

Minor and rare irAEs are hepatitis and myositis. Patients with immune-related hepatitis are asymptomatic and present with abnormal laboratory test results. In these Patients ultrasound or CT can show periportal edema, mild hepatomegaly and periportal lymphadenopathy $(2,13,25)$. However liver biopsy is necessary to make diagnosis of autoimmune hepatitis (31).

Contrast-enhanced CT can demonstrate new focal intramuscular enhancement, while PET/CT can show new intramuscular tracer uptake: these findings correlate with drug-related myositis (2).

\section{Future perspectives and challenges}

With the increasing role of immunotherapy in the treatment of lung cancer, diagnostic radiologists are asked to face increasingly difficult interpretative challenges: furthermore, in the next years the lung cancer clinical scenario will be even more complex.

Diagnostic radiologists will be asked to interpret the radiological response of combined therapies (multiple targeted therapies mixed up with conventional chemo regimens) and their potential interactions with locoregional therapies (radiation and interventional oncology) and their potential synergic and abscopal effect (32-34).

To overcome these difficulties, radiologists are asked to 
have a deep knowledge of the different classification criteria in order to correctly interpret the outcome of clinical trials.

Moreover, radiologists need to fully exploit the potential of the recent preliminary advances in the radiomics field (35-37), in order to better and deeply understand the clinical impact of the radiological responses after immunotherapy.

\section{Acknowledgements}

None.

\section{Footnote}

Conflicts of Interest: The authors have no conflicts of interest to declare.

\section{References}

1. Carter BW, Halpenny DF, Ginsberg MS, et al. Immunotherapy in Non-Small Cell Lung Cancer Treatment: Current Status and the Role of Imaging. J Thorac Imaging 2017;32:300-12.

2. Braschi-Amirfarzan M, Tirumani SH, Hodi FS, et al. Immune-Checkpoint Inhibitors in the Era of Precision Medicine: What Radiologists Should Know. Korean J Radiol 2017;18:42.

3. Wolchok JD, Hoos A, O'Day S, et al. Guidelines for the Evaluation of Immune Therapy Activity in Solid Tumors: Immune-Related Response Criteria. Clin Cancer Res 2009;15:7412-20.

4. Lim SH, Sun JM, Lee SH, et al. Pembrolizumab for the treatment of non-small cell lung cancer. Expert Opin Biol Ther 2016;16:397-406.

5. Nivolumab Approved for Lung Cancer. Cancer Discov 2015;5:OF1.

6. Weinstock C, Khozin S, Suzman D, et al. U.S. Food and Drug Administration Approval Summary: Atezolizumab for Metastatic Non-Small Cell Lung Cancer. Clin Cancer Res 2017;23:4534-9.

7. Hellmann MD, Ciuleanu TE, Pluzanski A, et al. Nivolumab plus Ipilimumab in Lung Cancer with a High Tumor Mutational Burden. N Engl J Med 2018. [Epub ahead of print].

8. Tirkes T, Hollar MA, Tann M, et al. Response Criteria in Oncologic Imaging: Review of Traditional and New Criteria. RadioGraphics 2013;33:1323-41.

9. Soria JC, Wu YL, Nakagawa K, et al. Gefitinib plus chemotherapy versus placebo plus chemotherapy in EGFR-mutation-positive non-small-cell lung cancer after progression on first-line gefitinib (IMPRESS): a phase 3 randomised trial. Lancet Oncol 2015;16:990-8.

10. Ferretti GR, Reymond E, Delouche A, et al. Personalized chemotherapy of lung cancer: What the radiologist should know. Diagn Interv Imaging 2016;97:287-96.

11. Shaw AT, Kim TM, Crinò L, et al. Ceritinib versus chemotherapy in patients with ALK -rearranged nonsmall-cell lung cancer previously given chemotherapy and crizotinib (ASCEND-5): a randomised, controlled, openlabel, phase 3 trial. Lancet Oncol 2017;18:874-86.

12. Nishino M, Giobbie-Hurder A, Gargano M, et al. Developing a Common Language for Tumor Response to Immunotherapy: Immune-Related Response Criteria Using Unidimensional Measurements. Clin Cancer Res 2013;19:3936-43.

13. Wang GX, Kurra V, Gainor JF, et al. Immune Checkpoint Inhibitor Cancer Therapy: Spectrum of Imaging Findings. RadioGraphics 2017;37:2132-44.

14. Khalil DN, Smith EL, Brentjens RJ, et Al. The future of cancer treatment: immunomodulation, CARs and combination immunotherapy. Nat Rev Clin Oncol 2016;13:273-90.

15. Chiou VL, Burotto M. Pseudoprogression and ImmuneRelated Response in Solid Tumors. J Clin Oncol 2015;33:3541-3.

16. Hodi FS, Hwu WJ, Kefford R, et al. Evaluation of Immune-Related Response Criteria and RECIST v1.1 in Patients with Advanced Melanoma Treated With Pembrolizumab. J Clin Oncol 2016;34:1510-7.

17. Tazdait M, Mezquita L, Lahmar J, et al. Patterns of responses in metastatic NSCLC during PD-1 or PDL-1 inhibitor therapy: Comparison of RECIST 1.1, irRECIST and iRECIST criteria. Eur J Cancer 2018;88:38-47.

18. Nishino M, Giobbie-Hurder A, Manos MP, et al. ImmuneRelated Tumor Response Dynamics in Melanoma Patients Treated with Pembrolizumab: Identifying Markers for Clinical Outcome and Treatment Decisions. Clin Cancer Res 2017;23:4671-9.

19. Carter BW, Bhosale PR, Yang WT. Immunotherapy and the role of imaging. Cancer 2018. [Epub ahead of print].

20. Kataoka Y, Hirano K, Narabayashi T, et al. Concordance between the response evaluation criteria in solid tumors version 1.1 and the immune-related response criteria in patients with non-small cell lung cancer treated with nivolumab: a multicenter retrospective cohort study. Cancer Chemother Pharmacol 2018;81:333-7. 
21. Seymour L, Bogaerts J, Perrone A, et al. iRECIST: guidelines for response criteria for use in trials testing immunotherapeutics. Lancet Oncol 2017;18:e143-52.

22. Nagai H, Muto M. Optimal management of immunerelated adverse events resulting from treatment with immune checkpoint inhibitors: a review and update. Int J Clin Oncol 2018;23:410-20.

23. El Osta B, Hu F, Sadek R, et al. Not all immunecheckpoint inhibitors are created equal: Meta-analysis and systematic review of immune-related adverse events in cancer trials. Crit Rev Oncol Hematol 2017;119:1-12.

24. Robert C, Schachter J, Long GV, et al. Pembrolizumab versus Ipilimumab in Advanced Melanoma. $\mathrm{N}$ Engl J Med 2015;372:2521-32.

25. Michot JM, Bigenwald C, Champiat S, et al. Immunerelated adverse events with immune checkpoint blockade: a comprehensive review. Eur J Cancer 2016;54:139-48.

26. Borghaei H, Paz-Ares L, Horn L, et al. Nivolumab versus Docetaxel in Advanced Nonsquamous Non-Small-Cell Lung Cancer. N Engl J Med 2015;373:1627-39.

27. Reck M, Rodríguez-Abreu D, Robinson AG, et al. Pembrolizumab versus Chemotherapy for PD-L1Positive Non-Small-Cell Lung Cancer. N Engl J Med 2016;375:1823-33.

28. Brahmer J, Reckamp KL, Baas P, et al. Nivolumab versus Docetaxel in Advanced Squamous-Cell Non-Small-Cell Lung Cancer. N Engl J Med 2015;373:123-35.

29. Nishino M, Ramaiya NH, Awad MM, et al. PD-1 Inhibitor-Related Pneumonitis in Advanced Cancer

Cite this article as: Calandri M, Solitro F, Angelino V, Moretti F, Veltri A. The role of radiology in the evaluation of the immunotherapy efficacy. J Thorac Dis 2018;10(Suppl 13):S1438-S1446. doi: 10.21037/jtd.2018.05.130
Patients: Radiographic Patterns and Clinical Course. Clin Cancer Res 2016;22:6051-60.

30. Delaunay M, Cadranel J, Lusque A, et al. Immunecheckpoint inhibitors associated with interstitial lung disease in cancer patients. Eur Respir J 2017;50:1700050.

31. Johncilla M, Misdraji J, Pratt DS, et al. Ipilimumabassociated Hepatitis: Clinicopathologic Characterization in a Series of 11 Cases. Am J Surg Pathol 2015;39:1075-84.

32. Mehta A, Oklu R, Sheth RA. Thermal Ablative Therapies and Immune Checkpoint Modulation: Can Locoregional Approaches Effect a Systemic Response? Gastroenterol Res Pract 2016;2016:9251375.

33. Palussière J, Catena V, Buy X. Percutaneous thermal ablation of lung tumors - Radiofrequency, microwave and cryotherapy: Where are we going? Diagn Interv Imaging 2017;98:619-25.

34. McArthur HL, Diab A, Page DB, et al. A Pilot Study of Preoperative Single-Dose Ipilimumab and/or Cryoablation in Women with Early-Stage Breast Cancer with Comprehensive Immune Profiling. Clin Cancer Res 2016;22:5729-37.

35. Colen RR, Fujii T, Bilen MA, et al. Radiomics to predict immunotherapy-induced pneumonitis: proof of concept. Invest New Drugs 2017. [Epub ahead of print].

36. Lambin P, Leijenaar RTH, Deist TM, et al. Radiomics: the bridge between medical imaging and personalized medicine. Nat Rev Clin Oncol 2017;14:749-62.

37. Wilson R, Devaraj A. Radiomics of pulmonary nodules and lung cancer. Transl Lung Cancer Res 2017;6:86-91. 